\title{
TIME VARIATIONS OF SOLAR X-RAY BRIGHT POINTS
}

\author{
L. GOLUB, A. S. KR IEGER, J. K. SILK, A. F. TIMOTHY \\ American Science \& Engineering, Cambridge, Mass., U.S.A.
}

and

G. S. VAIA NA

Center for Astrophysics, Cambridge, Mass., U.S.A.

Summary. An example of the overall view of the X-ray corona (nominal filter passband 2-32 $\AA$ and $44-54 \AA$ ) showing a coronal hole, filament activity, bright points and the large scale-scale loop structures, is shown in Figure 1. This is one of the 32000 X-ray images obtained with the AS \& E X-ray telescope on Skylab. A comprehensive review describing the characteristics of the various features and their implications regarding the high velocity solar streams, evolution of magnetic fields

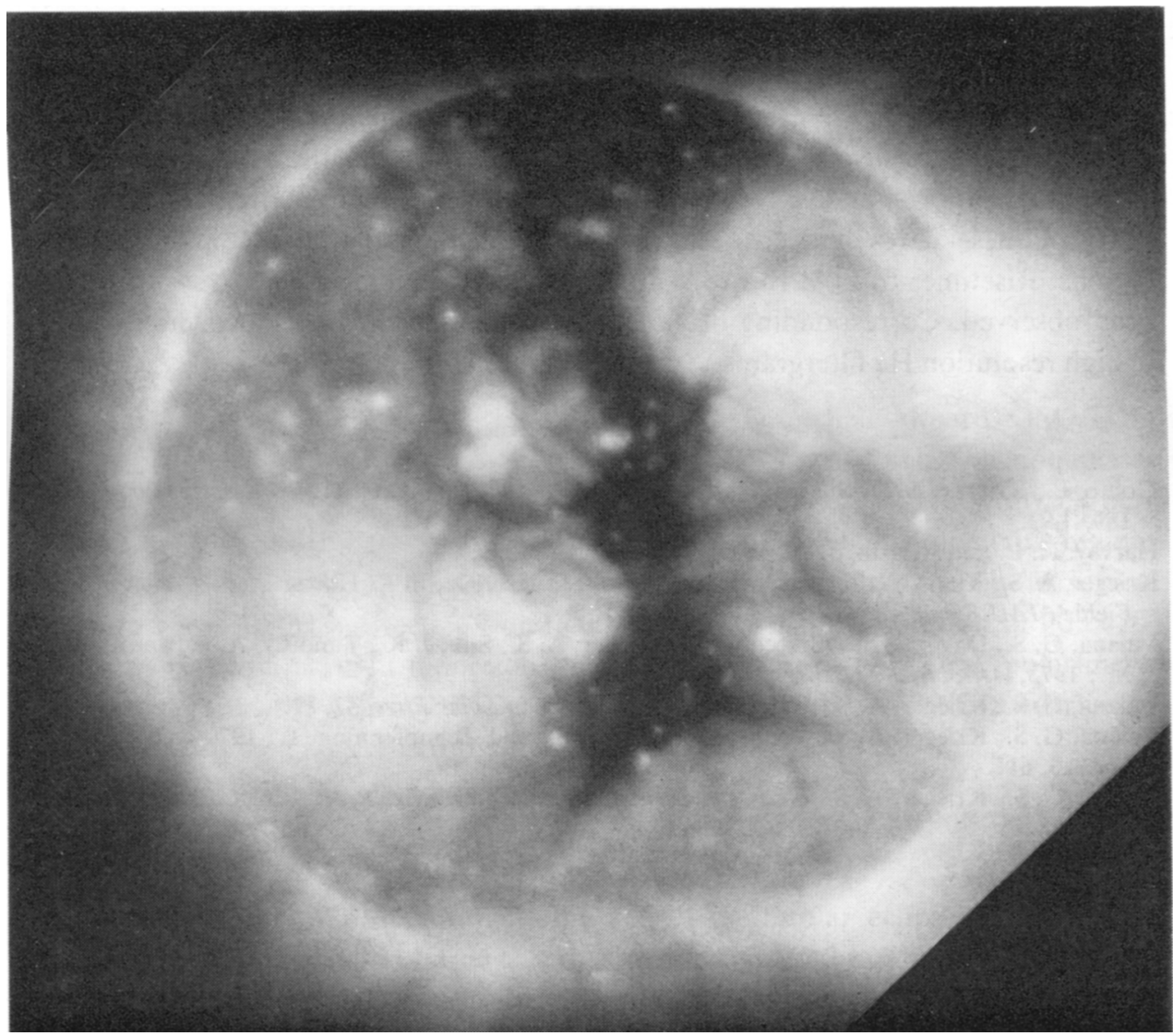

Fig. 1. Overall view of the X-ray corona on June 1, 1973 (nominal filter passband 2-32 $\AA$ and 44-45 $\AA$ ), showing a coronal hole, filament cavity, bright points and the large-scale loop structures. 
in active regions, and sources of soft X-ray emission has been given by Vaiana et al. (1975). In the present summary we will only be concerned with the bright points. Studies of solar X-ray bright points, show that these features represent a distinct class of solar activity. Bright points appear first as a diffuse cloud of soft X-ray emission typically growing to $30^{\prime \prime}$ in diameter, with growth rates of $\sim 1 \mathrm{~km} \mathrm{~s}^{-1}$. Several hours after the point first becomes visible a bright compact core forms, growing to $10^{\prime \prime}$. The lifetime distribution of bright points follows a Poisson distribution with a mean of eight hours (see references). The points are distributed uniformly over the entire solar surface, with approximately 500 on the Sun at any time. Their occurrrence appears to be independent of major active regions, except for a visibility factor near high loop structures or a possible decrease in number in active region latitudes.

Bright points show a one-to-one correspondence with bipolar magnetic features when comparison is made with simultaneous magnetograms having arcsec resolution and high sensitivity $(<10 \mathrm{G})$. Total flux values per point are $10^{19}-10^{20} \mathrm{Mx}$. There is also a strong correlation between $\mathrm{X}$-ray observations and those made in transition region and in chromospheric lines, such as the EUV lines or $\mathrm{Ca} \mathrm{K}$. However, the chromospheric observations show both bipolar and unipolar magnetic enhancements, so that X-ray photos are needed to identify the locations of bright points.

Bright points are also seen to undergo flare activity at all latitudes from the equator to the poles. Approximately $10 \%$ of all points flare during their lifetimes. A preliminary study of these flares indicates variability approaching that found in major active regions. Risetimes from $<10 \mathrm{~s}$ to $>10 \mathrm{~min}$ and decay times of $<1$ to $>30 \mathrm{~min}$ have been observed. Corresponding brightenings can be seen in chromospheric lines and in high resolution $\mathrm{H} \alpha$ filtergrams.

\section{References}

Golub, L., Krieger, A. S., Silk, J. K., Timothy, A. F., and Vaiana, G. S. : 1974, Astrophys. J. Letters 189, L93.

Harvey, K. L. and Martin, S. F.: 1973, Solar Phys. 32, 389.

Krieger, A. S., Viana, G. S., and Van Speybroeck, L. P.: 1971, in R. Howard (ed.), 'Solar Magnetic Fields', IAU Symp. 43, 397.

Vaiana, G. S., Davis, J. M., Giacconi, R., Krieger, A. S., Silk, J. K., Timothy, A. F., and Zombeck, M.: 1973, Astrophys. J. Letters 185, L47.

Vaiana, G. S., Krieger, A. S., and Timothy, A. F.: 1973, Solar Phys. 32, 81.

Vaiana, G. S., Krieger, A. S., Van Speybroeck, L. P., and Zehnpfenning, T.: 1970, Bull. Am. Phys. Soc. 15, 611 .

Vaiana, G. S., Krieger, A. S., Timothy, A. F., and Zombeck, M.: Proc. IAU Colloquium, No. 27, in press. 